\title{
A stiff-legged gait: benign acute childhood myositis
}

\section{Sangeeta Jain BMSc, Michael R. Kolber MD BSc}

Previously published at www.cmaj.ca

$\mathrm{A}$ previously healthy 6-year-old boy was brought to the emergency department with a sudden inability to walk or bear weight on his legs. Four days earlier, he had experienced a fever and runny nose and was given symptomatic treatment with simple analgesics at home. For the next two days, he had sporadically complained of "sore legs" and would transiently "toe-walk," only to subsequently take off running without any difficulty. On the morning of the fifth day of his illness, although his fever and coryza were improving, he had been unable to get out of bed. When his mother attempted to stand him up, he was unable to bear weight on his legs. He would ambulate only by crawling on all fours.

On presentation, the patient was afebrile and had normal vital signs. He complained of pain in both legs, pointing primarily to the area behind his knees. He was still unable to walk or bear weight. Physical examination showed normal strength in the lower extremities when the patient was lying down. The patients deep tendon reflexes were preserved both at L3/L4 and at L5/S1 and he had normal sensation bilaterally. Cranial nerves were intact. No rashes or signs of trauma to the lower limbs were found. Strength in the patient's upper extremities was normal and the rest of the physical examination was unremarkable.

Laboratory investigations showed normal leukocyte count (3.6 [normal 5-12] $\left.\times 10^{9} / \mathrm{L}\right)$, platelet count (151 [normal $\left.150-450] \times 10^{\circ} / \mathrm{L}\right)$ and erythrocyte sedimentation rate $(2$ [nor$\mathrm{mal}<10] \mathrm{mm} / \mathrm{h}$ ), but a markedly elevated serum creatinine kinase level of 3400 (normal 10-90) U/L. A urinalysis was normal, and radiographs of the pelvis, bilateral femurs and knees were normal with no evidence of fracture or malignancy. A nasopharyngeal viral swab was performed.

After consultation with the pediatric infectious disease service, we admitted the patient for observation and conservative management. The next day, the patient could take only two steps while two adults supported his weight. His gait, which $\vec{\circ}$ his mother described as a "Frankenstein walk," was stiff and broad-based.

On the third day after admission, the patient's condition rapidly improved. In a matter of hours, he progressed from being able to walk with support to an independent gait, and was discharged by noon of that day. The result of the nasopharyngeal swab, which was received after the patient

\section{Key points}

- Benign acute childhood myositis should be considered in children presenting with sudden gait-related abnormalities or refusal to bear weight after a viral illness, especially during influenza season.

- Boys from 3-14 years of age are most commonly affected.

- Neurologic findings are usually normal and creatinine kinase level is elevated.

- With conservative measures, the condition usually resolves spontaneously within a week and without residual sequelae. Rhabdomyolysis is a rare complication.

had been discharged, was positive for influenza B. The patient had completely recovered (i.e., was able to run) after about a week. He had no residual impairment and no recurrence of pain or weakness in the lower extremities. We concluded that the patient had a classic presentation of benign acute childhood myositis.

\section{Discussion}

Benign acute childhood myositis is known by various names, including influenza-associated myositis, viral myositis and acute myositis. We searched PubMed and MEDLINE using these terms and found a handful of case reports.

This disease was first described in a series of case reports by Lundberg in 1957, who studied 74 patients in Sweden with an illness he named "myalgia cruris epidemica". Seventy of these patients were school-aged children and most were boys. Since then, sporadic cases of benign acute childhood myositis have been reported worldwide, but the exact incidence and prevalence has not been established to date.

Benign acute childhood myositis tends to be more prevalent in the late winter and early spring, ${ }^{1-3}$ supporting the theory that its underlying cause is viral illness. ${ }^{2,4,5}$ As in our patient, most cases in countries of northern latitudes occur in March and April..$^{1-3}$ Generally, this condition affects school-

From the Department of Family Medicine, University of Alberta, Edmonton, Alta.

Cite as CMAJ 2009. DOI:10.1503/cmaj.090781 
Box 1: Differential diagnosis of benign acute childhood myositis $^{4,5,6,7,8}$

Guillain-Barré syndrome

- Symptoms present 2-4 weeks after viral illness

- Distal paresthesia and ascending paralysis

- Symmetric weakness with absent or decreased deep tendon reflexes

Deep vein thrombosis

- Associated with pre-existing malignancy, hypercoagulable states or central line insertion

- Unilateral pain, swelling and discoloration in limb

Osteomyelitis

- May have history of trauma to or penetration of skin

- Decreased movement, swelling of soft tissues with or without erythema in affected area

- Patient may present with malaise, fever and localized, unilateral pain

- Elevation of inflammatory markers (erythrocyte sedimentation rate, C-reactive protein, leukocyte count)

Juvenile rheumatoid arthritis

- Subacute presentation over months

- Swelling and tenderness in joints that is worse in the morning

Fracture

- History of trauma, unilateral symptoms

- Abnormal radiographic findings

Malignancy

- Presentation is subacute, with associated fever, weight loss and bone pain that is worse at night

- Hematologic (e.g., with complete blood count, erythrocyte sedimentation rate) and radiographic abnormalities are generally present

aged children, primarily boys, within the age range of 3-14 years old. ${ }^{5,6}$

Many viral agents have been linked with benign acute childhood myositis; however, influenza A and B have been most frequently associated with the disease. Of the two main isolated strains of influenza, influenza B appears to be responsible for more cases than influenza A. ${ }^{5}$

\section{Clinical presentation}

The typical clinical scenario begins with a prodromal illness lasting an average of five days and including fever, flu-like symptoms and symptoms of infection of the upper respiratory tract. ${ }^{4-6}$ The onset of myositis generally becomes apparent as the prodromal illness, particularly fever, subsides. ${ }^{46}$ Patients typically present with acute bilateral pain or weakness in the lower legs that results in refusal or inability to bear weight after a period of rest, such as on awakening. ${ }^{6}$

Two gait-related abnormalities were reported by Mackay and colleagues ${ }^{6}$ to be characteristic of benign acute childhood myositis: a wide-based, stiff-legged gait and toe-walking. Both abnormalities were present in our patient. This abnormality is due to pain and discomfort in the lower extremities, commonly in the gastrocnemius-soleus muscle group bilaterally. ${ }^{4,6}$ Cases have been reported in which other muscle groups were affected (e.g., upper arms), but this finding is relatively rare and invariably associated with symptoms in the lower limbs. ${ }^{5}$ Physical examination generally shows normal strength, tone and deep tendon reflexes in the lower extremities and no neurologic deficits.

\section{Investigations}

Typical laboratory values in benign acute childhood myositis include a markedly elevated serum creatinine kinase level,,${ }^{46}$ which tends to resolve within one to two weeks. Commonly, patients have leukopenia as well as increases in aspartate aminotransferase and alanine aminotransferase levels.

\section{Pathophysiology}

The exact mechanism by which myositis occurs is controversial. Current theories are that damage occurs via immunemediated processes or that the virus particles themselves invade the muscle tissue to cause damage. ${ }^{5}$ The second theory is supported by studies in which researchers have isolated viral particles from biopsies of calf muscle in children with suspected benign acute childhood myositis. ${ }^{5}$ Generally, such biopsies show nonspecific degenerative changes and muscle necrosis. ${ }^{5}$

\section{Differential diagnosis}

The differential diagnosis in pediatric populations for failure to weight-bear includes Guillain-Barré syndrome, osteomyelitis, deep vein thrombosis, juvenile rheumatoid arthritis, fracture or malignancy (Box 1). ${ }^{5}$

Guillain-Barré syndrome, the most important differential diagnosis, typically presents with an abnormal neurologic examination, with absent or decreased deep tendon reflexes in the lower extremities. ${ }^{5}$ Fracture and osteomyelitis are generally unilateral in presentation and may have associated radiographic findings, whereas malignancy and juvenile rheumatoid arthritis typically present subacutely with a predominantly asymmetric distribution. Finally, deep vein thrombosis may also be unilateral and is relatively rare in otherwise healthy pediatric populations. ${ }^{5,7}$

\section{Complications}

A rare complication of benign acute childhood myositis is rhabdomyolysis resulting in kidney damage secondary to myoglobinemia. ${ }^{5,8}$ Clinicians should be aware of this rare but serious complication and look for signs of rhabdomyolysis, including dark urine that dips positive for blood but does not show red blood cells under microscopy. Rhabdomyolysis is more common in girls with benign acute childhood myositis. ${ }^{8}$ 


\section{Prevention}

The association between vaccination status against influenza and risk of benign acute childhood myositis is unknown. Our patient had not received the influenza vaccine during the year when he had benign acute childhood myositis. Although patients who have received the influenza vaccine may be assumed to be at lower risk for common post-influenza complications (e.g., lower respiratory infections and acute otitis media), a systematic review failed to show a reduction in secondary complications with the vaccine. ${ }^{9}$

This article has been peer reviewed.

Competing interests: None declared.

Contributors: Michael Kolber was the attending physician for the clinical case. Both of the authors were involved in the conception of the article and the review and interpretation of the literature, and both of them drafted the manuscript and approved the final version submitted for publication.

\section{REFERENCES}

1. Lundberg A. Myalgia Cruris Epidemica. Acta Paediatr 1957;46:18-31.

2. Middleton PJ, Alexander RM, Szymanski MT. Severe myositis during recovery from influenza. Lancet 1970;2:533-5

3. Dietzman DE, Schaller JG, Ray G, et al. Acute myositis associated with influenza B infection. Pediatrics 1976;57:255-8.

4. Zafeiriou DI, Katzos G, Gombakis N, et al. Clinical features, laboratory findings and differential diagnosis of benign acute childhood myositis. Acta Paediatr 2000;89:1493-4.

5. Agyeman P, Duppethaler A, Heininger U, et al. Influenza-associated myositis in children. Infection 2004;32:199-203

6. Mackay MT, Kornberg AJ, Sheila LK, et al. Benign acute childhood myositis: laboratory and clinical features. Neurology 1999;53:2127-31.

7. Müjgen Sonmez F, Cakir M, Yayla S, et al. Benign acute childhood myositis. Med Princ Pract 2004;13:227-9.

8. King BA. Benign acute childhood myositis as a cause of failure to weight bear. $J$ Paediatr Child Health 2003;39:378-80.

9. Jefferson T, Smith S, Demicheli V, et al. Assessment of the efficacy and effectiveness of influenza vaccines in healthy children: systematic review. Lancet 2005;365:773-80.

Correspondence to: Dr. Michael R. Kolber, Department of Family Medicine, University of Alberta, Box 7590, Peace River AB

T8S 1T2; fax 780624 4015; mkolber@ualberta.ca

The section Cases presents brief case reports that convey clear, practical lessons. Preference is given to common presentations of important rare conditions, and important unusual presentations of common problems. Articles start with a case presentation (500 words maximum), and a discussion of the underlying condition follows (1000 words maximum). Generally, up to 5 references are permitted and visual elements (e.g., tables of the differential diagnosis, clinical features or diagnostic approach) are encouraged. Written consent from patients for publication of their story is a necessity and should accompany submissions. See information for authors at www.cmaj.ca

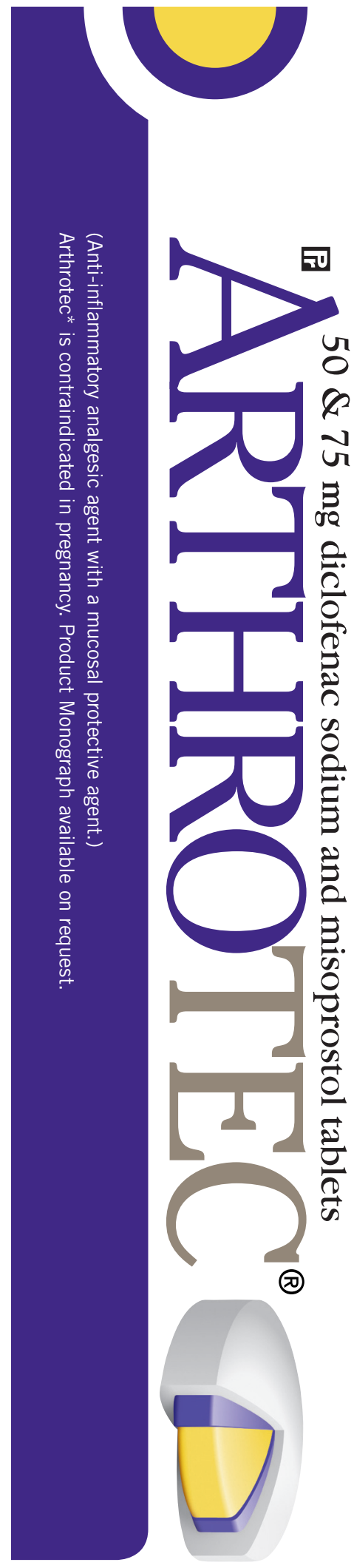

